\title{
Subspecialized radiological reporting reduces radiology report turnaround time
}

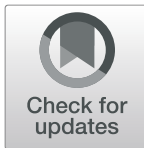

Andreas Otto Josef Zabel ${ }^{1,2^{*}}$ (D), Sebastian Leschka ${ }^{1,2}$, Simon Wildermuth ${ }^{1,2}$, Juerg Hodler ${ }^{2,3}$ and Tobias Johannes Dietrich ${ }^{1,2}$

\begin{abstract}
Objectives: The objective of this study was to compare the radiology report turnaround time (RTAT) between decentralized/modality-based and centralized/subspecialized radiological reporting at a multi-center radiology enterprise.
\end{abstract}

Methods: RTAT values for MRI, CT, and conventional radiography were compared between decentralized/ modality-based (04 September 2017-22 December 2017) and centralized/subspecialized radiology (03 September 2018-21 December 2018) reporting grouped into three subspecializations (body radiology, musculoskeletal radiology, and neuroradiology) at eleven sites of a multi-center radiology enterprise. For the objective of this investigation, hospitals were defined as major and minor hospitals. The Mann-Whitney $U$ test served for statistical analyses.

Results: Change of reporting system from decentralized/modality-based to centralized/subspecialized radiology resulted overall in a significant decrease of the RTAT: from 82 to $77 \mathrm{~min}$ for the first signature $(p<0.001)$, and 119 to $107 \mathrm{~min}$ and 295 to $238 \mathrm{~min}$ for the second signature $(p<0.001)$. Subgroup analyses demonstrate a significant decrease of the RTAT for MRI reports (e.g., second signature RTAT, 1051 to $401 \mathrm{~min} ; p<0.001$ ) and conventional radiographs (e. g., second signature RTAT, 278 to $171 \mathrm{~min} ; p<0.001$ ). The RTAT at major hospitals decreased from 288 to 245 min (second signature; $p<0.001$ ) while the corresponding RTAT of minor hospitals decreased more remarkably, from 300 to $198 \mathrm{~min}(p<0.001)$. However, the results were heterogenous; in some analyses, the RTAT even increased. The effect size analyses represent small effects.

Conclusions: Change of reporting system from decentralized/modality-based to centralized/subspecialized radiology was associated with a significant decreased RTAT. Specifically, the RTAT for MRI reports and conventional radiographs was significantly reduced. A pronounced RTAT decrease was observed at minor hospitals.

Keywords: Specialization, Change management, Process assessment, health care

\footnotetext{
* Correspondence: andreas.zabel@kssg.ch

'Division of Radiology and Nuclear Medicine, Kantonsspital St. Gallen,

Rorschacherstrasse 95, 9007 St. Gallen, CH, Switzerland

${ }^{2}$ Faculty of Medicine, University of Zurich, Pestalozzistrasse 3, CH-8091 Zürich,

Switzerland

Full list of author information is available at the end of the article
}

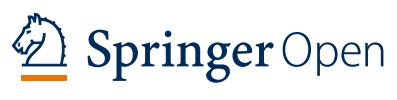

(c) The Author(s). 2020 Open Access This article is licensed under a Creative Commons Attribution 4.0 International License, which permits use, sharing, adaptation, distribution and reproduction in any medium or format, as long as you give appropriate credit to the original author(s) and the source, provide a link to the Creative Commons licence, and indicate if changes were made. The images or other third party material in this article are included in the article's Creative Commons licence, unless indicated otherwise in a credit line to the material. If material is not included in the article's Creative Commons licence and your intended use is not permitted by statutory regulation or exceeds the permitted use, you will need to obtain permission directly from the copyright holder. To view a copy of this licence, visit http://creativecommons.org/licenses/by/4.0/. 


\section{Key points}

- Changing the reporting system can result in a reduced report turnaround time (RTAT).

- RTAT decreased especially for MRI reports and conventional radiographs.

- RTAT of minor hospitals decreased more remarkably compared with major hospitals.

\section{Introduction}

Neuroradiology and pediatric radiology are established subspecializations within the radiology community. In daily practice, further subspecialization is more common in the USA [1] than in most European radiology departments [2]. Subspecialized radiology has been implemented in academic centers [3] but also at larger private and public institutions [1]. Growing radiology networks and teleradiology services are major trends in radiology, which have resulted in significant changes in radiology [4].

Utilization management of radiological manpower may be more efficient in radiology networks than in smaller units of radiology operating in a number of different hospitals. Furthermore, higher imaging volumes permit the development of subspecialists within one radiology network [5]. Therefore, radiology networks may increase the proportion of subspecialty-focused radiology reporting. Our radiology network consists of eleven radiological sites of various sizes across the entire country (Fig. 1). In the beginning, radiologists were sent to each of the radiological sites and worked in the hospitals using the radiology information system (RIS) for reporting. They were supported by other radiologists using teleradiology. Between 2017 and 2018, a paradigm shift from decentralized/modality-based to centralized/subspecialized reporting was performed. Thereby most of the radiologists were transferred to the main hospital and subspecialization groups were formed while a small number of radiologists remained at the other radiological sites for modality-specific problems and on-site services such as interaction with patients and staff at the hospitals. All imaging studies were divided into different subspecializations and mainly reported by the radiologists within the individual group. We hypothesized that changing the reporting system from general to subspecialized radiological reporting associated with centralization of radiologists reduces the radiology report turnaround time (RTAT).

The aim of this study was to compare the RTAT between decentralized/modality-based and centralized/ subspecialized radiological reporting.

\section{Materials and methods Radiology network}

The radiology network consists of eleven radiology sites of various sizes, providing radiology services as a part of primary and secondary health care centers for a population of 500,000 individuals (December 2018) [6]. Moreover, the main general hospital provides tertiary health care services for more than 1,000,000 individuals. The setup of the radiology network and the included imaging studies are illustrated in Fig. 1. Major hospitals were defined as hospitals with more than 5000 reports during the examined periods and the availability of at least one MR scanner. The four major hospitals providing inpatient and outpatient medical service are equipped with digital radiography and CT and MR scanners. During the evaluation period of 2018, a second MR scanner was put into service in major hospital \#2 (Fig. 1) in addition to the MR scanner already in place since 2017. The six minor hospitals that did not fulfill the two major criteria are providing inpatient and outpatient medical service. They are equipped with digital radiography and a CT scanner but not with a MR scanner. In addition, there is one outpatient medical center in the investigated network equipped with one MR scanner and one PET/CT, which did not fulfill the criteria for neither major nor minor hospitals as it is a dedicated outpatient medical center and not a hospital. The PET/CT is occasionally used for the acquisition of standard CT images only,

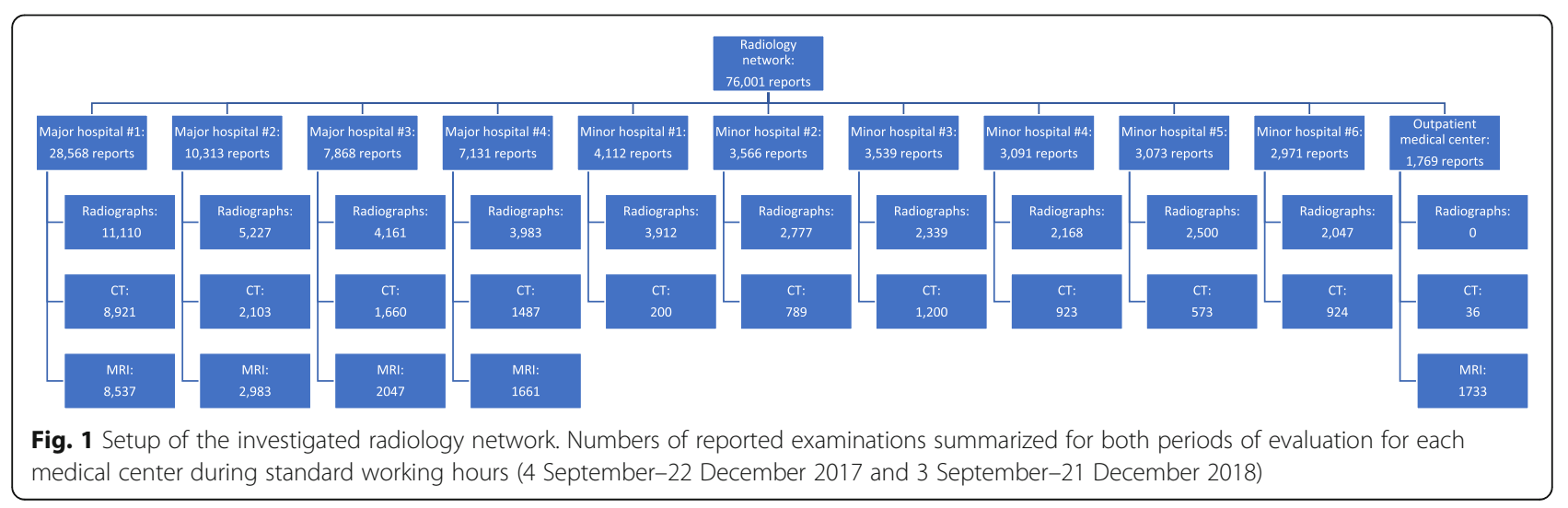


without positron emission tomography modality. The radiology department includes a radiology resident training program. During both investigated periods, as part of the real-life setting, residents got board-certified and new residents were hired. However, there was an unchanged number of residents and board-certified radiologists for both evaluation periods. Residents were trained at the main hospital but could rotate between the several major and minor hospitals. The same RIS (Ana+ 2.3.6879.18755, Cobra Software AG, Arlesheim, Switzerland), PACS (IMPAX EE R20 XVIII v20190821_ 0813, AGFA HealthCare N. V., Mortsel, Belgium), voice recognition software (Dragon Medical Direct 5.0 (19.1.4741.0), Nuance Communications, Inc, Burlington, MA, USA) [7, 8], and standardized PACS workstations/ Hardware and standardized protocols were employed throughout the entire study, both before and after the paradigm shift from decentralized/modality-based to centralized/subspecialized organization.

\section{Reorganization from modality-/center-based to subspecialized reporting}

Until 7 January 2018, the common practice was a decentralized/modality-based workflow with board-certified radiologists or residents responsible for all imaging studies and imaging modalities at each specific site or for a certain imaging modality (radiographs, fluoroscopy, CT, MRI, or ultrasound). Starting in January 2018, the decentralized/modality-based workflow was replaced by centralized radiology with subspecialized teams, supported by teleradiology. The imaging studies of all modalities of the eleven radiology sites were sorted into four subspecializations (body radiology, musculoskeletal radiology, neuroradiology, and pediatric radiology) with each team led by fellowship-trained radiologists. Residents were to be trained in the subspecialized teams and rotated every 6 months. Board-certified radiologists focused on one of the four subspecializations. Reports were acquired by an unchanged number of residents and board-certified radiologists for both periods of evaluation.

The first 9 months (January-September 2018) were considered as a transitional period between both periods of evaluation during which radiologists would adopt the new subspecialization system. Teleradiology-based reporting across the eleven sites was applied to balance radiology report workload among the radiologists at various sites.

\section{Outcome measures}

RTATs were determined by subtracting the time from image acquisition from the first and second electronic signatures of the reports, respectively. Radiology reports can exclusively be viewed by the clinically working doctors after the final signature. Experienced residents were authorized to sign final reports for radiographs but required a final signature from a board-certified radiologist for CT and MRI reports. Board-certified radiologists signed final reports for all imaging modalities but had the opportunity to consult with a senior radiologist for a second signature. RTATs were extracted from the integrated RIS-/PACS database (76,001 reports total). Data analyses of the present study exclusively included radiology examinations performed during core working hours on weekdays (Monday-Friday: 6:54 AM to 16:59 PM) between 4 September 2017 and 22 December 2017 for the decentralized/modality-based system and between 3 September 2018 and 21 December 2018 for the centralized/subspecialized system. Weekends, public holidays, and night shifts were excluded as subspecialized reporting was not feasible due to reduced staffing outside standard working hours. The workflow shift from decentralized/modality-based to centralized/subspecialized radiology reporting did not influence the workflow for nuclear medicine, pediatric radiology, and interventional radiology including CT-guided procedures, fluoroscopy, ultrasound, or mammography. Thus, these procedures and examinations were excluded from the analysis of the present study.

\section{Statistical analysis}

The Kolmogorov-Smirnov test and Q-Q plots indicated that the RTAT values were not normally distributed [9]. Thus, the RTAT values are demonstrated as medians (= 50th percentiles) and 80th percentiles. The 80th percentile RTAT was chosen to determine medium-challenging/ time-consuming reports. The Mann-Whitney $U$ test served for statistical comparison of RTAT values between decentralized/modality-based radiology system (04 September 2017-22 December 2017) and centralized/subspecialized radiology system (03 September 2018-21 December 2018). The $p$ value was adjusted according to the Bonferroni correction [10] because data of the present study underwent multiple testing; thus, a $p<0.00121(=0.05 / 41$, number of tests $n=41)$ was considered to denote a statistical significant difference between both periods of evaluation. According to Cohen's guidelines of Pearson's $r$, effect size $(r)$ values smaller than 0.3 were considered to represent a small effect, values between 0.3 and 0.5 were considered to represent a medium effect, and values larger than 0.5 were considered to represent a large effect [11]. Subgroup analyses evaluated imaging modalities such as MRI, CT, and conventional radiographs; subspecializations; and major and minor hospitals. Furthermore, the turnaround time for reports written by residents and board-certified radiologists was compared. Statistical analyses were 
performed using SPSS (version 25.0.0.1, IBM Corporation, Armonk, NY, USA).

\section{Results}

During the first evaluation period (04 September 201722 December 2017), 37,247 imaging examinations (radiography, CT, MRI) were reported by the decentralized/ modality-based radiology system during standard working hours. This was compared to 38,754 reports which were acquired during the second evaluation period (03 December 2018-21 December 2018) by the centralized/ subspecialized system (3.9\% increase). The number of reports with two signatures decreased from 16,632 to 15, 967 (4.0\% decrease) between the first and second evaluation periods.

\section{Overall turnaround time}

The median RTAT for the first signature was 32 min for both evaluation periods. The 80th percentile RTAT, however, decreased $(p<0.001, r=0.02)$ from 82 to 77 min (Table 1), and the median RTAT for the second signature also decreased ( $p<0.001, r=0.06)$, from 119 to $107 \mathrm{~min}$, between the two periods. The corresponding 80th percentile RTAT decreased from 295 to $238 \mathrm{~min}$.

\section{Turnaround time for imaging modalities}

Subgroup analyses demonstrate a significant difference $(p<0.001)$ of the RTAT between the two evaluation periods for all evaluated imaging modalities (Table 2).

For both first and second signatures, the median RTAT (2017, 27 and $117 \mathrm{~min}$; 2018, 20 and $77 \mathrm{~min}$ ) and 80th percentile RTAT (2017, 71 and $278 \mathrm{~min}$; 2018, 51 and $171 \mathrm{~min})$ decreased $(p<0.001, r=0.14$ and 0.21 , respectively) for conventional radiographs.

Between the two periods, the median RTAT increased $(p<0.001, r=0.09)$ for the first signature on MRI reports; by contrast, the 80th percentile RTAT for the first signature on MRI reports decreased from 124 to 112 min. Both the median and 80th percentile RTAT for the second signature on MRI reports decreased $(p<0.001, r$ = 0.09; median 206 to $163 \mathrm{~min}$; 80th percentile, 1051 to $401 \mathrm{~min}$ ), while the median RTAT and 80th percentile RTAT for both signatures on CT reports increased $(p<$ $0.001, r=0.09$ and 0.15 , respectively).
Report turnaround time for subspecialization reporting Subgroup analyses demonstrate a difference $(p<0.001)$ of the RTAT between the two evaluation periods for all evaluated subspecializations (Table 3 ).

The median and 80th percentile RTAT, for both signatures on body radiology reports, increased $(p<0.001, r$ $=0.04$ and 0.05 , respectively).

For first and second signatures on musculoskeletal radiology reports, the median RTAT (2017, 30 and 123 min; 2018, 26 and $81 \mathrm{~min}$ ) and 80th percentile RTAT (2017, 80 and $300 \mathrm{~min} ; 2018,64$ and $170 \mathrm{~min}$ ) decreased ( $p<0.001, r=0.06$ and 0.21 , respectively).

Both the median and 80th percentile RTAT for the first signature on neuroradiology reports increased $(p<$ $0.001, r=0.15)$. The median RTAT increased for the second signature on neuroradiology reports; by contrast, the 80th percentile RTAT for the second signature decreased from 409 to $346 \min (p<0.001, r=0.05)$.

\section{Comparison of major versus minor hospitals}

The outpatient medical center did not fulfill the criteria for neither major nor minor hospitals. Thus, the imaging examinations were excluded from the comparison of major versus minor hospitals.

\section{Overall turnaround time of major versus minor hospitals}

Table 4 shows both the median and 80th percentile RTAT for the first signature at the major hospitals increased $(p<0.001, r=0.02)$. By contrast, both the median and 80th percentile RTAT for the first signature at the minor hospitals decreased $(p<0.001, r=0.12$; median 32 to $24 \mathrm{~min}$, 80th percentile 92 to $62 \mathrm{~min}$ ). At both major and minor hospitals, for the second signature, both the median and 80th percentile RTAT decreased ( $p$ $<0.001, r=0.03$ and 0.15 , respectively). The 80th percentile RTAT decreased at major hospitals from 288 to $245 \mathrm{~min}$ and decreased at minor hospitals from 300 to $198 \mathrm{~min}$.

\section{Turnaround time for imaging modalities of major versus minor hospitals}

Subgroup analyses show a difference for the RTAT of the evaluated imaging modalities at major and minor hospitals between the first and second evaluation periods (Table 4).

Table 1 Overall radiology report turnaround time of all hospitals —04 September 2017-22 December 2017 vs. 03 September $2018-$ 21 December 2018

\begin{tabular}{|c|c|c|c|c|c|c|c|}
\hline \multirow[b]{2}{*}{ Period } & \multicolumn{2}{|c|}{ Number } & \multicolumn{2}{|c|}{ Median [min] } & \multicolumn{2}{|c|}{ 80th percentile $[\mathrm{min}]$} & \multirow[t]{2}{*}{$p$ value ( $r$ value) } \\
\hline & 2017 & 2018 & 2017 & 2018 & 2017 & 2018 & \\
\hline 1st signature & 37,247 & 38,754 & 32 & 32 & 82 & 77 & $<.001^{*}(r=0.02)$ \\
\hline 2nd signature & 16,632 & 15,967 & 119 & 107 & 295 & 238 & $<.001 *(r=0.06)$ \\
\hline
\end{tabular}

min minutes. *Significant $p$ value 
Table 2 Comparison of radiology report turnaround time by modalities (all hospitals) —04 September 2017-22 December 2017 vs. 03 September 2018-21 December 2018

\begin{tabular}{|c|c|c|c|c|c|c|c|}
\hline \multirow[b]{2}{*}{ Period } & \multicolumn{2}{|c|}{ Number } & \multicolumn{2}{|c|}{ Median [min] } & \multicolumn{2}{|c|}{ 80th percentile [min] } & \multirow[t]{2}{*}{$p$ value ( $r$ value) } \\
\hline & 2017 & 2018 & 2017 & 2018 & 2017 & 2018 & \\
\hline \multicolumn{8}{|l|}{ MRI } \\
\hline 1st signature & 8122 & 8839 & 45 & 55 & 124 & 112 & $<.001 *(r=0.09)$ \\
\hline 2nd signature & 3837 & 4581 & 206 & 163 & 1051 & 401 & $<.001^{*}(r=0.09)$ \\
\hline \multicolumn{8}{|l|}{ CT } \\
\hline 1st signature & 9058 & 9758 & 35 & 41 & 78 & 90 & $<.001 *(r=0.09)$ \\
\hline 2nd signature & 4547 & 4688 & 87 & 110 & 168 & 214 & $<.001^{*}(r=0.15)$ \\
\hline \multicolumn{8}{|c|}{ Conventional radiographs } \\
\hline 1st signature & 20,067 & 20,157 & 27 & 20 & 71 & 51 & $<.001 *(r=0.14)$ \\
\hline 2nd signature & 8248 & 6698 & 117 & 77 & 278 & 171 & $<.001 *(r=0.21)$ \\
\hline
\end{tabular}

Most interestingly, the 80th percentile RTAT of the second signature on conventional radiographs decreased at major hospitals from 248 to $156 \mathrm{~min}$, while it decreased from 331 to $189 \mathrm{~min}$ at minor hospitals $(p<0.001, r=0.21$ and 0.23 , respectively).

Turnaround time for subspecialization reporting of major versus minor hospitals

Subgroup analyses demonstrate a difference between the RTAT of the evaluated subspecializations at major and minor hospitals between the first and second evaluation periods (Table 5).

Most interestingly, the median and 80th percentile RTAT for musculoskeletal radiology reports decreased for both signatures at major $(p<0.001, r=0.02$ and 0.20 , respectively) and minor $(p<0.001, r=0.12$ and 0.24 , respectively) hospitals (80th percentile: major hospitals from 269 to $157 \mathrm{~min}$, minor hospitals from 344 to $192 \mathrm{~min}$ ).
Turnaround time for residents versus board-certified radiologists

Interestingly, subgroup analyses demonstrate a difference between the RTAT of reports written by boardcertified radiologists between the first and second evaluation periods (Table 6). For the first signature, the median RTAT decreased from 29 to $27 \mathrm{~min}$ and the 80th percentile RTAT decreased from 80 to $69 \mathrm{~min}(p<$ $0.001, r=0.05)$ between the two periods. For the second signature, the median RTAT decreased from 119 to 107 min, while the corresponding 80th percentile RTAT decreased from 296 to $238 \mathrm{~min}(p<0.001, r=0.06)$.

By contrast, there was no significant difference between the median and 80th percentile RTAT of reports written by residents $(p<0.013, r=0.01)$ between the two evaluation periods.

\section{Discussion}

The most important finding of this study is that changing the reporting system from decentralized/modality-

Table 3 Comparison of radiology report turnaround time by subspecializations (all hospitals) — 04 September 2017-22 December 2017 vs. 03 September 2018-21 December 2018

\begin{tabular}{|c|c|c|c|c|c|c|c|}
\hline \multirow[b]{2}{*}{ Period } & \multicolumn{2}{|c|}{ Number } & \multicolumn{2}{|c|}{ Median [min] } & \multicolumn{2}{|c|}{ 80th percentile [min] } & \multirow[t]{2}{*}{$p$ value ( $r$ value) } \\
\hline & 2017 & 2018 & 2017 & 2018 & 2017 & 2018 & \\
\hline \multicolumn{8}{|l|}{ Body radiology } \\
\hline 1st signature & 14,259 & 14,211 & 35 & 33 & 86 & 89 & $<.001 *(r=0.04)$ \\
\hline 2nd signature & 6969 & 5428 & 113 & 128 & 252 & 267 & $<.001 *(r=0.05)$ \\
\hline \multicolumn{8}{|c|}{ Musculoskeletal radiology } \\
\hline 1st signature & 16,392 & 17,718 & 30 & 26 & 80 & 64 & $<.001 *(r=0.06)$ \\
\hline 2nd signature & 6419 & 6898 & 123 & 81 & 300 & 170 & $<.001 *(r=0.21)$ \\
\hline \multicolumn{8}{|l|}{ Neuroradiology } \\
\hline 1st signature & 6596 & 6825 & 31 & 44 & 79 & 85 & $<.001 *(r=0.15)$ \\
\hline 2nd signature & 3244 & 3641 & 128 & 139 & 409 & 346 & $<.001 *(r=0.05)$ \\
\hline
\end{tabular}

min minutes. *Significant $p$ value 
Table 4 Comparison of major vs. minor hospitals' radiology report turnaround time by modalities — 04 September 2017-22 December 2017 vs. 03 September 2018-21 December 2018

\begin{tabular}{|c|c|c|c|c|c|c|c|c|c|c|c|c|c|c|}
\hline \multicolumn{8}{|l|}{ Major hospitals } & \multicolumn{7}{|c|}{ Minor hospitals } \\
\hline & \multicolumn{2}{|l|}{$n$} & \multicolumn{2}{|c|}{$\begin{array}{l}\text { Median } \\
\text { [min] }\end{array}$} & \multicolumn{2}{|c|}{$\begin{array}{l}\text { 80th } \\
\text { percentile } \\
\text { [min] }\end{array}$} & \multirow[t]{2}{*}{$p$ value ( $r$ value) } & \multicolumn{2}{|l|}{$n$} & \multicolumn{2}{|c|}{$\begin{array}{l}\text { Median } \\
\text { [min] }\end{array}$} & \multicolumn{2}{|c|}{$\begin{array}{l}\text { 80th } \\
\text { percentile } \\
\text { [min] }\end{array}$} & \multirow[t]{2}{*}{$p$ value ( $r$ value) } \\
\hline Period & 2017 & 2018 & 2017 & 2018 & 2017 & 2018 & & 2017 & 2018 & 2017 & 2018 & 2017 & 2018 & \\
\hline \multicolumn{15}{|l|}{ All modalities } \\
\hline 1st signature & 26,524 & 27,356 & 32 & 34 & 78 & 81 & $<.001 *(r=0.02)$ & 9906 & 10,446 & 32 & 24 & 92 & 62 & $<.001^{*}(r=0.12)$ \\
\hline 2nd signature & 13,195 & 11,868 & 117 & 110 & 288 & 245 & $<.001 *(r=0.03)$ & 3270 & 3663 & 125 & 91 & 300 & 198 & $<.001 *(r=0.15)$ \\
\hline \multicolumn{15}{|l|}{$\mathrm{CT}$} \\
\hline 1st signature & 6897 & 7274 & 33 & 42 & 71 & 92 & $<.001 *(r=0.14)$ & 2150 & 2459 & 40 & 38 & 108 & 83 & $.01 *(r=0.04)$ \\
\hline 2nd signature & 3799 & 3677 & 86 & 109 & 162 & 212 & $<.001 *(r=0.16)$ & 746 & 997 & 93 & 111 & 204 & 224 & $<.001^{*}(r=0.09)$ \\
\hline \multicolumn{15}{|c|}{ Conventional radiographs } \\
\hline 1st signature & 12,311 & 12,170 & 26 & 19 & 63 & 49 & $<.001 *(r=0.13)$ & 7756 & 7987 & 30 & 20 & 88 & 54 & $<.001 *(r=0.16)$ \\
\hline 2nd signature & 5724 & 4032 & 110 & 73 & 248 & 156 & $<.001 *(r=0.21)$ & 2524 & 2666 & 137 & 83 & 331 & 189 & $<.001 *(r=0.23)$ \\
\hline
\end{tabular}

min minutes, $C T$ computed tomography. *Significant $p$ value

based radiology to centralized/subspecialized radiology resulted in a significant decrease of the RTAT. Overall, the RTAT decreased from 82 to 77 min (80th percentile) for the first signature $(p<0.001)$, while it decreased from 119 to $107 \mathrm{~min}$ (median) and from 295 to $238 \mathrm{~min}$ (80th percentile) for the second signature $(p<0.001)$. Subgroup analyses demonstrated a significant decrease of the RTAT for MRI reports (e.g., second signature, 80th percentile RTAT, 1051 to $401 \mathrm{~min} ; p<0.001$ ) and conventional radiographs (e. g., second signature, 80th percentile RTAT, 278 to $171 \mathrm{~min} ; p<0.001$ ).

It has to be noted that the effect size analyses correspond to small effects. Notably, minor hospitals benefited most from the change to centralized/subspecialized radiology, as the RTAT decreased overall from 300 to 198 min (second signature, 80th percentile; $p<0.001$ ), while the corresponding RTAT of major hospitals decreased, less remarkably, from 288 to $245 \mathrm{~min}(p<0.001)$.

Similarly, to other recently published surveys, the present study demonstrated an annual increase of 3.9\% in the number of radiology reports and imaging examinations during the evaluation period in 2018 compared to 2017 (Table 1) [3, 12, 13]. Interestingly, there was an increase from 37,247 to 38,754 reports but a decrease in reports with a second signature from 16,632 to 15,967 reports (Table 1). During the period of decentralized/ modality-based radiology, radiologists occasionally forwarded difficult reports to specialists for a second opinion and a second signature, respectively. One may speculate that centralized/subspecialized radiology

Table 5 Comparison of major vs. minor hospitals' radiology report turnaround time by subspecializations —04 September 2017-22 December 2017 vs. 03 September 2018-21 December 2018

\begin{tabular}{|c|c|c|c|c|c|c|c|c|c|c|c|c|c|c|}
\hline \multirow[b]{3}{*}{ Period } & \multicolumn{7}{|c|}{ Major hospitals } & \multicolumn{7}{|c|}{ Minor hospitals } \\
\hline & \multicolumn{2}{|l|}{$n$} & \multicolumn{2}{|c|}{$\begin{array}{l}\text { Median } \\
\text { [min] }\end{array}$} & \multicolumn{2}{|c|}{$\begin{array}{l}\text { 80th } \\
\text { percentile } \\
\text { [min] }\end{array}$} & \multirow[t]{2}{*}{$p$ value ( $r$ value) } & \multicolumn{2}{|l|}{$n$} & \multicolumn{2}{|c|}{$\begin{array}{l}\text { Median } \\
\text { [min] }\end{array}$} & \multicolumn{2}{|c|}{$\begin{array}{l}\text { 80th } \\
\text { percentile } \\
\text { [min] }\end{array}$} & \multirow[t]{2}{*}{$p$ value ( $r$ value) } \\
\hline & 2017 & 2018 & 2017 & 2018 & 2017 & 2018 & & 2017 & 2018 & 2017 & 2018 & 2017 & 2018 & \\
\hline \multicolumn{15}{|l|}{ Body radiology } \\
\hline 1st signature & 10,722 & 10,591 & 35 & 36 & 83 & 94 & $.46(r=0.01)$ & 3368 & 3342 & 34 & 25 & 93 & 66 & $<.001^{*}(r=0.15)$ \\
\hline 2nd signature & 5821 & 4295 & 115 & 131 & 256 & 277 & $<.001 *(r=0.06)$ & 1114 & 1001 & 105 & 107 & 231 & 217 & $.61(r=0.01)$ \\
\hline \multicolumn{15}{|c|}{ Musculoskeletal radiology } \\
\hline 1st signature & 10,042 & 10,915 & 29 & 27 & 74 & 66 & $<.001 *(r=0.02)$ & 6096 & 6553 & 31 & 23 & 94 & 59 & $<.001 *(r=0.12)$ \\
\hline 2nd signature & 4374 & 4343 & 114 & 79 & 269 & 157 & $<.001 *(r=0.20)$ & 1997 & 2464 & 145 & 84 & 344 & 192 & $<.001 *(r=0.24)$ \\
\hline \multicolumn{15}{|l|}{ Neuroradiology } \\
\hline 1st signature & 5760 & 5850 & 30 & 44 & 77 & 86 & $<.001 *(r=0.16)$ & 442 & 551 & 26 & 31 & 78 & 65,6 & $.22(r=0.04)$ \\
\hline 2nd signature & 3000 & 3230 & 131 & 141 & 423 & 346 & $<.001^{*}(r=0.05)$ & 159 & 198 & 73 & 106 & 147 & 185 & $.004(r=0.15)$ \\
\hline
\end{tabular}

min minutes. *Significant $p$ value 
Table 6 Comparison of radiology report turnaround time residents versus board-certified radiologists—04 September 2017-22 December 2017 vs. 03 September 2018-21 December 2018

\begin{tabular}{|c|c|c|c|c|c|c|c|}
\hline \multirow[b]{2}{*}{ Period } & \multicolumn{2}{|c|}{ Number } & \multicolumn{2}{|c|}{ Median [min] } & \multicolumn{2}{|c|}{ 80th percentile [min] } & \multirow[t]{2}{*}{$p$ value ( $r$ value) } \\
\hline & 2017 & 2018 & 2017 & 2018 & 2017 & 2018 & \\
\hline \multicolumn{8}{|l|}{ Residents } \\
\hline 1st signature & 17,037 & 18,109 & 36 & 37 & 85 & 86 & $.013(r=0.01)$ \\
\hline \multicolumn{8}{|c|}{ Board-certified radiologists } \\
\hline 1st signature & 20,132 & 20,584 & 29 & 27 & 80 & 69 & $<.001 *(r=0.05)$ \\
\hline 2nd signature & 16,551 & 15,862 & 119 & 107 & 296 & 238 & $<.001 *(r=0.06)$ \\
\hline
\end{tabular}

increases the experience and confidence of radiologists in their dedicated tasks. Thus, centralized/subspecialized radiology may decrease the necessity for requesting a second opinion for challenging radiology reports. The increasing number of radiology reports of $3.9 \%$ between the evaluation periods 2017 and 2018 can be considered as the normal annual growth rate of our radiology department.

Thus, as hypothesized, it appears that the RTAT decreased in spite of the increased workload. One may assume that the experience gained by residents and board-certified radiologists may also have improved the RTAT values. Nonetheless, new residents were hired and residents got board-certified as part of the real-life setting. Nonetheless, there was an unchanged number of residents and board-certified radiologists for both evaluation periods. Interestingly, after changing the reporting system from decentralized/modality-based radiology to centralized/subspecialized radiology, the RTAT for reports written by board-certified radiologists decreased significantly, while there was no significant difference of the RTAT for reports written by residents (Table 6). As board-certified radiologists unlike residents focus on one of the four subspecializations, it may also indicate an improvement between the two investigated systems of radiological reporting.

Recent literature revealed conflicting data regarding the RTAT: Change of reporting system from decentralized/modality-based radiology to centralized/subspecialized radiology demonstrated both a decrease of the RTAT, as shown by the results of Stern et al. [14], and, conversely, an increase of the RTAT, as shown by the results of Meyl et al. [3]. In the present study, the change to a centralized/subspecialized system of radiological reporting leads overall to a significant decrease of the RTAT (Tables 1 and 4). The RTAT for MRI reports and conventional radiographs decreased most significantly (Tables 2 and 4), which other studies confirm [14]. In some important and critical areas, significant increases of the RTAT were noted, e.g., for the median of the first signature of MR reports (Table 2), for both signatures of body radiology (Table 3), for the first signature of neuroradiology (Table 3), and for the first signature of major hospitals (Table 4). This may be explained by redistributive effects. Not all areas of this heterogeneous survey benefited from the system change to centralized/ subspecialized radiological reporting.

Before the change management from decentralized/ modality-based radiology to centralized/subspecialized radiology, the radiology network showed a very low RTAT (Table 1) compared with peer-valued studies [14], which could be improved even further after the change (Table 1). Interestingly, there was an increase for both signatures of the RTAT for CT reports (Table 2) which may be explained because of its very complex or time-consuming cases, by a work redistribution in favor of MRI reports and by general redistribution effects from the management. Time-critical CT reports (e.g., stroke, trauma) were given higher priority than MR reports or reports for conventional radiographs in both evaluated systems of radiological reporting. In addition, a senior staff radiologist was in duty to identify time-sensitive examinations and, if necessary, to distribute them along the radiologists to provide fast reports for emergency and urgent cases. However, this study did not differentiate between emergency and routine $\mathrm{CT}$ examinations.

In contrast to the results of Meyl et al. [3], the current study showed particularly that the RTAT for mediumchallenging/time-consuming reports, represented by the 80th percentile RTAT, decreased significantly; this is especially true of the RTAT for the subspecialization of musculoskeletal radiology. In the other subspecializations, however, the trend tended towards increased RTATs (Tables 3 and 5), which was also observed by other recent studies [3].

Furthermore, as suspected, there was a connection between the size of hospitals and change of reporting system. Notably, minor hospitals noted a general improvement of the RTAT overall, as well as a significant improvement of the RTAT for conventional radiographs and CT scans (Table 4). Furthermore, minor hospitals benefited from a decreased RTAT for the body and musculoskeletal subspecializations (Table 5). The 
decreased RTAT may demonstrate a better distribution of workload among all radiologists through the use of teleradiology, in spite of the more centralized and subspecialized multi-center radiology network [15-17].

The findings of this study differ from the other cited surveys, as it provides RTATs not only for single academic or public hospitals but for a multi-center radiology network consisting of eleven radiology siteswhich represent public hospitals of various sizes (Fig. 1)-as well as a fully integrated diagnostic neuroradiology department, which is an unusual setup compared to most larger European radiology departments [2].

The limitations of this statistical evaluation were many confounding variables including the experience of radiologists, case complexity, and case volume. Furthermore, as part of the real-life setting, the number of studies included by modality was not equitable as the number of conventional radiographs exceeds the number of CT and MRI reports. Although RTAT may not be considered as the best measure to determine efficiency of a radiology department, it is a frequently used scale for the evaluation of the workflow of many radiology institutions [18-21] as well as the 80th percentile RTAT [14, 22, 23]. Outlier (= very complex or time-consuming cases) such as cardiovascular CT or MR examinations were difficult to define. However, these examinations occurred in both systems of radiological reporting and are part of everyday clinical practice in large institutions. Regarding distortion caused by outliers, the median serves as a robust measurement [24]. According to Cohen's guidelines, most results of the study show a small effect size. However, there are indications to consider these normative guidelines and to use correlations of $0.10,0.20$, and 0.30 to represent relatively small, typical, and relatively large effects [25].

A study comparing the quality of radiology reports between decentralized/modality-based radiology and centralized/subspecialized radiology was conducted and will be published as a separate study.

In conclusion, changing the reporting system from decentralized/modality-based radiology to a centralized/ subspecialized radiology was associated with a significant decrease of the RTAT overall and a significant decrease of the RTAT for MRI reports and conventional radiographs. A significant decrease of the RTAT at minor hospitals, organized in a multi-center radiology network, was also noted-a significant improvement between the two evaluation periods. The effect size corresponds to small effects.

\section{Abbreviations}

$\mathrm{CT}$ : Computed tomography; MRI: Magnetic resonance imaging; PACS: Picture archiving and communication system; PET/CT: Positron emission tomography CT; RIS: Radiological information system; RTAT: Radiology report turnaround time

\section{Authors' contributions}

AZ was a major contributor in conceptualization, methodology, software, validation, formal analysis, investigation, resources, data curation, writing, original draft preparation, visualization, and project administration.

SL was a major contributor in conceptualization, methodology, writing, review, editing, and supervision.

SW was a major contributor in conceptualization, methodology, writing, review, editing, and supervision.

$\mathrm{JH}$ was a major contributor in conceptualization, methodology, writing, review, editing, and supervision.

TD was a major contributor in conceptualization, methodology, software, validation, formal analysis, investigation, resources, data curation, writing, original draft preparation, visualization, and project administration.

The authors read and approved the final manuscript.

\section{Funding}

This research did not receive any specific grant from funding agencies in the public, commercial, or not-for-profit sectors.

\section{Availability of data and materials}

The full SPSS datasets can be uploaded if it is desired.

\section{Ethics approval and consent to participate}

The study protocol was submitted to the local institutional review board. The local institutional review board declared that according to current law neither institutional review board approval nor informed consent was required.

\section{Consent for publication \\ Not applicable.}

\section{Competing interests}

The authors declare that they have no competing interests.

\section{Author details}

${ }^{1}$ Division of Radiology and Nuclear Medicine, Kantonsspital St. Gallen, Rorschacherstrasse 95, 9007 St. Gallen, CH, Switzerland. 'Faculty of Medicine, University of Zurich, Pestalozzistrasse 3, CH-8091 Zürich, Switzerland.

${ }^{3}$ Department of Diagnostic and Interventional Radiology, University Hospital of Zurich, Rämistrasse 100, 8091 Zürich, CH, Switzerland.

Received: 28 July 2020 Accepted: 30 September 2020

Published online: 30 October 2020

\section{References}

1. Rosenkrantz AB, Wang W, Hughes DR, Duszak R Jr (2018) Generalist versus subspecialist characteristics of the U.S. radiologist workforce. Radiology 286 : 929-937

2. Rehani B, Zhang YC, Rehani MM et al (2017) Radiology education in Europe: analysis of results from 22 European countries. World J Radiol. 9:55-62

3. Meyl TP, de Bucourt M, Berghöfer A et al (2019) Subspecialization in radiology: effects on the diagnostic spectrum of radiologists and report turnaround time in a Swiss university hospital. Radiol Med. 124:860-869

4. Fleishon HB, Vijayasarathi A, Pyatt R, Schoppe K, Rosenthal SA, Silva E 3rd. (2019) White paper: corporatization in radiology. J Am Coll Radiol. 16:13641374

5. European Society of Radiology (2010) The future role of radiology in healthcare. Insights Imaging. 1:2-11

6. Swiss Federal Statistical Office - STAT-TAB, online database - Ständige und nichtständige Wohnbevölkerung nach institutionellen Gliederungen, Geburtsort und Staatsangehörigkeit (in German)

7. Glaser C, Trumm C, Nissen-Meyer S, Francke M, Küttner B, Reiser M (2005) Speech recognition: impact on workflow and report availability. Radiologe. 45:735-742

8. Boland GW, Guimaraes AS, Mueller PR (2008) Radiology report turnaround: expectations and solutions. Eur Radiol. 18:1326-1328

9. Altman DG, Bland JM (1995) Statistics notes: the normal distribution. BMJ. 310:298

10. Victor A, Elsässer A, Hommel G, Blettner M (2010) Judging a plethora of pvalues: how to contend with the problem of multiple testing--part 10 of a series on evaluation of scientific publications. Dtsch Arztebl Int. 107:50-56 
11. Cohen J (1992) A power primer. Psychol Bull. 112:155-159

12. Oechtering TH, Panagiotopoulos N, Völker M et al (2020) Work and training conditions of German residents in radiology - results from a nationwide survey conducted by the young radiology forum in the German Roentgen Society. Rofo. 192:458-470

13. Forsberg D, Rosipko B, Sunshine JL (2017) Radiologists' variation of time to read across different procedure types. J Digit Imaging. 30:86-94

14. Stern C, Boehm T, Seifert B, Kawel-Boehm N (2018) Subspecialized radiological reporting expedites turnaround time of radiology reports and increases productivity. Rofo. 190:623-629

15. Dionisio JD, Taira RK, Sinha U et al (2000) Teleradiology as a foundation for an enterprise-wide health care delivery system. Radiographics. 20:1137-1150

16. Brand J, Bernegger A, Pressinger D, Schindler M, Neubauer T (2019) Multihospital use of imaging techniques in decentralized trauma care. Unfallchirurg. 122:328-332

17. Bradley WG Jr (2012) Teleradiology. Neuroimaging Clin N Am. 22:511-517

18. Rao SH, Virarkar M, Yang WT et al (2020) Streamlining the quantitative metrics workflow at a comprehensive cancer center. Acad Radiol S10766332(20)30364-30360. doi:https://doi.org/10.1016/j.acra.2020.06.014

19. Baccei SJ, Henderson SR, Lo HS, Reynolds K (2020) Using quality improvement methodology to reduce costs while improving efficiency and provider satisfaction in a busy, academic musculoskeletal radiology division. J Med Syst. 44:104

20. Seithe T, de Bucourt M, Busse R et al (2015) Teleradiological report turnaround times: an internal efficiency and quality control analysis. Radiologe 55(5):409-416

21. Rathnayake S, Nautsch F, Goodman TR, Forman HP, Gunabushanam G (2017) Effect of radiology study flow on report turnaround time. AJR Am J Roentgenol. 209:1308-1311

22. Prevedello LM, Ledbetter S, Farkas C, Khorasani R (2014) Implementation of speech recognition in a community-based radiology practice: effect on report turnaround times. J Am Coll Radiol. 11:402-406

23. Andriole KP, Prevedello LM, Dufault A et al (2010) Augmenting the impact of technology adoption with financial incentive to improve radiology report signature times. J Am Coll Radiol. 7:198-204

24. Kwak SK, Kim JH (2017) Statistical data preparation: management of missing values and outliers. Korean J Anesthesiol. 70:407-411

25. Brydges CR (2019) Effect size guidelines, sample size calculations, and statistical power in gerontology. Innov Aging. 4;3(4):igz036

\section{Publisher's Note}

Springer Nature remains neutral with regard to jurisdictional claims in published maps and institutional affiliations.

\section{Submit your manuscript to a SpringerOpen ${ }^{\circ}$ journal and benefit from:}

- Convenient online submission

- Rigorous peer review

- Open access: articles freely available online

- High visibility within the field

- Retaining the copyright to your article

Submit your next manuscript at $\boldsymbol{\nabla}$ springeropen.com 